\title{
Kalkschulter per Ultraschall gezielt aufspüren und Schmerzen mit Stoßwellen lindern
}

Berlin - Wenn die Schulter in der Nacht schmerzt und das vom Schlafen abhält oder Haare kämmen nahezu unmöglich erscheint - dann kann eine Kalkschulter die Ursache sein. Bei Kalkansammlungen, die starke Schmerzen verursachen, wird den Patienten manchmal zur Operation geraten. Eine schonende Alternative dazu kann die extrakorporale Stoßwellentherapie (ESWT) sein. Dabei richtet der behandelnde Arzt mit einem Ultraschallgerät energiereiche Stoßoder Druckwellen gezielt auf die betroffene Schulter. Und auch bei der Diagnose kann das Ultraschallverfahren sehr hilfreich sein. Wie die Diagnostik und die Stoßwellentherapie genau funktionieren und warum sie so erfolgsversprechend sind, erläutert Dr. Rainer Berthold, Experte der Deutschen Gesellschaft für Ultraschall in der Medizin e. V. (DEGUM).

Bei einer Kalkschulter bilden sich Kalkansammlungen in einer Sehne in der Schulter. Ursache können mechanische Faktoren sowie lokale Durchblutungs- oder Stoffwechselstörungen sein. „Bewegungen über Kopf, aber auch nach hinten oder zur Seite mit Belastung sind äußerst schmerzhaft", so beschreibt Dr. Rainer Berthold, einer der stellvertretenden DEGUM-Sektionsleiter Chirurgie, die Symptome einer Kalkschulter. Die Vielfalt der Beschwerden lässt die Kalkschulter als Chamäleon erscheinen. Wegen der starken Schmerzen können die Patienten den Arm oft kaum noch bewegen und nicht auf der betroffenen Schulter liegen; durch eine Schonhaltung kann es sogar zur Versteifung der Schulter kommen.

Von einer Kalkschulter sind vor allem Menschen zwischen 35 und 50 Jahren betroffen, rund 2 Drittel davon sind Frauen. Die Erkrankung wird meistens erst recht spät diagnostiziert, denn solange die Ablagerungen klein sind, verursachen sie keine Symptome. Es ist davon auszugehen, dass ein asymptomatischer Kalkherd zu etwa 40 Prozent im weiteren Verlauf zu Beschwerden führt. „Wir können die Verdachtsdiagnose, die wir aufgrund des Beschwerdebildes und der Bewegungseinschränkung gewonnen haben, mithilfe des Ultraschallverfahrens und einer Röntgenuntersuchung bestätigen“, erklärt Dr. Berthold. „Diese Kombination ist im Vergleich zur Kernspintomografie von hoher Treffsicherheit und sie ist schnell und kostengünstig durchführbar.“

Warum sich die Kalkansammlungen bilden, ist noch nicht endgültig geklärt. Eine Ursache könnte eine Mangeldurchblutung und damit ein Sauerstoffmangel in den Schultersehnen sein. Ein therapeutischer Ansatz ist deshalb die Aktivierung des Stoffwechsels und somit auch der Durchblutung - durch gezielte Bewegung. Spezielle Dehnübungen helfen, den Schmerz zu lindern. Bei Bedarf verordnet der Arzt zudem noch Medikamente gegen Schmerzen oder Entzündungen, um die Patienten, die häufig im Alltag und im Beruf stark eingeschränkt sind, kurzfristig zu entlasten. Manchmal setzen auch Physiotherapeuten therapeutischen Ultraschall unterstützend zur Schmerzbehandlung ein.

Solange die Patienten mit ihren Beschwerden gut zurechtkommen, kann der meist gutartige Spontanverlauf der Erkrankung abgewartet werden. Denn oft lösen sich die Verkalkungen im Laufe der Zeit wieder von allein auf - das kann aber Monate dauern und ist mit starken Schmerzen verbunden. Bei großen Kalkansammlungen wird den Patienten manchmal zur Operation geraten. Eine schonende Alternative dazu kann jedoch die Stoßwellentherapie sein. Der Nutzen der Behandlung in Bezug auf eine Besserung der Symptome und Verkleinerung der Kalkdepots ist inzwischen in vielen Studien nachgewiesen. Dabei setzen Ärzte das Ultraschallverfahren nicht nur zur Diagnose, sondern auch zur präziseren Behandlung ein.

Bei der fokussierten extrakorporalen Stoßwellentherapie (ESWT) werden mithilfe der sogenannten Piezoelektrik außerhalb des
Körpers Impulse erzeugt, die innerhalb des Körpers wirksam werden. Bei der Therapie der Kalkschulter richtet der Arzt fokussierte Stoßwellen gezielt auf das Kalkdepot in der Schultersehne. Dieser Fokus lässt sich berechnen und das Gerät je nach Position des Kalkdepots adaptieren. Die Stoßwellenwirkung kann dann exakt auf die gewünschte Zone ausgerichtet werden. Das Gewebe um die Kalkansammlung - also die Haut, die Muskulatur und das Bindegewebe wird somit nicht beeinträchtigt. „Oft wird angenommen, die Stoßwellen würden das Kalkdepot zerstören. Dies ist aber nicht richtig - vielmehr bewirkt der Druckimpuls die Induktion zellulärer Reaktionen“, erklärt Dr. Berthold. „Die vermehrte Durchblutung mit Gefäßneubildungen im betroffenen Areal führt danach zur Auflösung der Kalkdepots."

Alternativ gibt es die kostengünstigere radiale Stoßwellentherapie. Sie arbeitet meist mit Druckluft. Durch den Aufschlag eines Projektils auf einen Applikator werden hier die nicht fokussierten Druckwellen erzeugt. Allerdings ist die Effektivität dieses niederenergetischen Verfahrens geringer - und die Anzahl der notwendigen Behandlungen damit meist höher.

Die Anwendung gehört für gesetzlich Versicherte zu den individuellen Gesundheitsleistungen (IGeL); der Patient muss die Kosten selbst tragen. Die meisten privaten Kassen und Beihilfestellen übernehmen die Behandlung. „1 bis 3 Behandlungen, die jeweils etwa 10 Minuten dauern, reichen bei der fokussierten Stoßwelle meistens aus“, versichert Dr. Berthold. „Die Nebenwirkungen sind gering, die Patienten werden durch dieses schonende Verfahren häufig wieder schmerzfrei und in der Schulter beweglich. Die Krankheitsdauer wird verkürzt und eine Operation ist in der Regel vermeidbar. Die ESWT lässt sich mit vorheriger Lokalisierung des Kalkdepots mittels Sonografie nach meiner Erfahrung exakter durchführen." 